\title{
COBERTURAS MEDIÁTICAS, POLARIZACIÓN Y REFORMAS EDUCATIVAS EN EsPaña
}

\author{
Media Coverage, Polarization and Educational Reforms in Spain
}

\section{IGNACIO-JESÚS SERRANO-CONTRERAS}

Universidad de Granada, España

\section{JAVIER GARCÍA-MARÍN}

Universidad de Granada, España

\author{
ÓSCAR G. LUENGO \\ Universidad de Granada, España
}

\begin{abstract}
RESUMEN
Las leyes de educación comportan, para todos los partidos políticos, una de las correas transmisoras de su proyecto ideológico. De este modo, cada uno de los diferentes gobiernos españoles, como ocurre en otros países, han llevado a cabo modificaciones legislativas con el fin de implementar políticas de un signo más cercano a sus intereses partidistas. A su vez, el contexto mediático, siguiendo los postulados de los académicos en comunicación política, ha tendido a apoyar $\mathrm{u}$ oponerse a dichas reformas, generando una escena polarizada. La presente investigación realiza un análisis del panorama español, durante los últimos cuarenta años, sobre los impactos que las reformas educativas han suscitado en el ámbito mediático. Se emplean para ello nuevas metodologías de aprendizaje de máquina (machine learning), complementadas con el desarrollo de una serie de medidores diseñados para captar fenómenos sociales complejos dentro de escenarios de variabilidad semántica. Los resultados han evidenciado una clara relación entre partidos políticos y medios, así como el posicionamiento que estos últimos, en consonancia con su línea editorial, mostraron con respecto a las reformas educativas que se iban sucediendo a lo largo del periodo de estudio.
\end{abstract}

Palabras clave: sistemas mediáticos, cobertura mediática, reformas educativas, polarización, machine learning

\begin{abstract}
Education laws involve, for all political parties, one of the most visible flagships of their ideological project. In this regard, different governments have made legislative modifications to introduce orientations in this field closer to their partisan interests. Therefore, following existing models of political communication, in Southern European countries the coverage of this information leads to a potential dichotomized media context. The present research displays an analysis of the Spanish case during the last forty years, to verify the influence that educational reforms have provoked in the press coverage. For that purpose, we have used new machine learning methodologies, complemented by the development of self measures, to capture complex social phenomena within semantic variability scenarios. As a result of those processes, findings have evidenced a clear relationship between the ideological proposal of political parties and the editorial line of the printed press, regarding the mentioned educational reforms that were developed under the period of study.
\end{abstract}

Keywords: Media Systems, Media Coverage, Educational Reforms, Polarization, Machine Learning 


\section{INTRODUCCIÓN}

La educación es uno de los ámbitos de actuación estratégica más importantes, sensibles y potencialmente conflictivos para los poderes públicos o, en palabras de Gil et al. (1998) una inversión estratégica de futuro. En la mayoría de los casos, marca la línea ideológica de los gobiernos en el poder, siendo un espacio de política pública donde no siempre es fácil encontrar el consenso entre los principales partidos políticos y, por lo tanto, se hace extremadamente complicado el objetivo de implementar políticas de estado que puedan soportar y sobrevivir a cambios de gobierno y de ciclo económico. El sector educativo es un terreno especialmente abonado para el desarrollo y defensa de posiciones beligerantes en materia religiosa, lingüística, identitaria e ideológica en España (Gortázar y Moreno 2017).

Desde los años 80, y con especial intensidad en esa década, hemos pasado por un periodo de reformas en el ámbito educativo español que han afectado a todos los niveles de formación: a protagonistas y responsables, a los estudiantes $\mathrm{y}$ al profesorado, o a la situación laboral de estos últimos. Aunque las primeras reformas encontraron como elemento dinamizador la reflexión y la voluntad de cambio, consolidando así el gran paradigma de la transición política, los hechos han terminado conformando una senda marcada por la gran cantidad de cambios normativos, estructurales, curriculares, organizativos y administrativos. El caso español ha sido señalado por los expertos como un ejemplo complejo donde se han sucedido reformas que, en muchos casos, han sido abortadas, frustradas, torpedeadas o abandonadas como consecuencia de cambios políticos, incluso dentro del mismo gobierno o del mismo partido (Viñao 2006: 50). De este modo, ha terminado por imponerse, como tónica general en las distintas legislaturas, una deriva sustentada en la propuesta y aprobación de nuevas leyes educativas.

Así, tras la aprobación de la constitución de 1978, se han sucedido en España casi una decena de reformas educativas a varios niveles, impulsadas por distintos partidos políticos que ostentaban el poder, y que proponían cambios de mayor o menor calado en el marco regulatorio vigente. En 1980, bajo el gobierno de la desaparecida UCD (Unión de Centro Democrático) se aprobó la LOECE ${ }^{1}$, que nunca entró en vigor. El Gobierno socialdemócrata presidido por Felipe González (PSOE, Partido Socialista Obrero Español) impulsó la Ley de Reforma Universitaria (LORU²) en 1983, la $\operatorname{LODE}^{3}$ (1985) y, posteriormente, la LOG$\mathrm{SE}^{4}$ (1990). El Partido Popular (PP) ratificó la Ley de Universidades $\left(\mathrm{LOU}^{5}\right)$ en 2001, la Ley de Formación Profesional ${ }^{6}$ y la $\mathrm{LOCE}^{7}$ en 2002. De nuevo el PSOE

\footnotetext{
Ley Orgánica 5/1980, de 19 de junio, por la que se regula el Estatuto de Centros Escolares. Ley Orgánica 11/1983, de 25 de agosto, de Reforma Universitaria. Ley Orgánica 8/1985, de 3 de julio, reguladora del Derecho a la Educación.

Ley Orgánica 1/1990, de 3 de octubre, de Ordenación General del Sistema Educativo. Ley Orgánica 6/2001, de 21 de diciembre, de Universidades. Ley Orgánica 5/2002, de 19 de junio, de las Cualificaciones y de la Formación Profesional. Ley Orgánica 10/2002, de 23 de diciembre, de Calidad de la Educación.
} 
en 2006 consigue aprobar la $\mathrm{LOE}^{8}$, ampliada un año más tarde para derogar la LOU $^{9}$. A la última gran reforma educativa vigente hasta 2020, la $\mathrm{LOMCE}^{10}$ de 2013, promovida por el gobierno conservador del PP, le han sucedido nuevos cambios con la tramitación en noviembre de 2020 de la denominada "Ley Celaá" (LOMLOE) ${ }^{11}$, que está generando las mismas discrepancias que sus predecesoras (y que, por su reciente aprobación, no se incluye en este estudio).

Todos estos modelos políticos sobre la educación y la contundencia de los cambios o las estrategias, han impulsado o limitado la fragmentación de su tramitación parlamentaria, lo cual podría interpretarse como un indicador de polarización política. A la vista de las votaciones la aritmética parlamentaria refleja que la polarización mayor fue la referida a la LOMCE (con el Partido Popular como único partido a favor, con 182 votos a favor, y 130 votos en contra de más de 5 grupos parlamentarios) ${ }^{12}$, siendo además promulgada bajo un clima de gran contestación social en las calles.

Las reformas educativas de magnitud han venido reflejando presiones sociales, culturales, económicas, ideológicas, históricas, e incluso religiosas. Se constituye así un panorama con intereses y visiones difícilmente coincidentes sobre el mundo del conocimiento, la educación y sus funciones (Monarca et al. 2016). A los procesos macro le acompañan otro tipo de dinámicas más cercanas que pueden generar resistencia a las reformas y, consecuentemente, polarización (González y Bouza 2009), como la oposición gremial, la carencia de apoyos sociales o políticos, los diagnósticos erróneos, la falta de dotación presupuestaria, los objetivos y calendarios irreales, la premura (Viñao 2006), o el posicionamiento ideológico de los medios de comunicación; su beligerancia o actitud proactiva. Son estos últimos los que se revelan como el principal escenario donde se mantienen y escenifican los asuntos públicos (Bennett et al 2004), además de un agente capaz de influir tanto en los actores políticos como en los ciudadanos (Collins y Cooper 2012).

\section{PLURALISMO POLARIZADO}

Las disensiones ideológicas que presentan la promulgación de cambios educativos se ven también extrapoladas a la arena mediática. Las líneas editoriales de los medios se transforman en un factor donde la neutralidad periodística se pone en tela de juicio, con lo que dejan a un lado su papel de contrapoder (Balkin 1999). Conocedores los medios del influyente rol que desempeñan en

Ley Orgánica 2/2006, de 3 de mayo, de Educación.

Ley Orgánica 4/2007, de 12 de abril, por la que se modifica la Ley Orgánica 6/2001, de 21 de diciembre, de Universidades.

10 Ley Orgánica 8/2013, de 9 de diciembre, para la Mejora de la Calidad Educativa.

11 Ley Orgánica 3/2020, de 29 de diciembre, por la que se modifica la Ley Orgánica 2/2006, de 3 de mayo, de Educación.

12 Partido Socialista Obrero Español, Convergencia i Unió, Izquierda Unida, Unión Progreso y Democracia, Partido Nacionalista Vasco y Grupo Mixto. 
la confección de políticas públicas (algo que confirman las teorías de agenda, por ejemplo), se alinean en una posición estratégica con la que establecen tanto a unos receptores subjetivos y contextualizados (Arriagada et al. 2010; Lodola y Kitzberger 2017), como a un encuadre específico para el mensaje (Entman 1993).

Siguiendo el modelo de Hallin y Mancini (2004), los países incluidos en el pluralismo polarizado se caracterizan, entre otras cosas, por la militancia política de sus medios de comunicación, esto es, por su paralelismo político (Humanes et al. 2013). Es de esperar entonces que, en periodos de reformas educativas, las posiciones expresadas por los principales partidos políticos; tanto por el que presenta el proyecto de ley, como por el que ejerce el papel de oposición, sean asumidas por los principales medios de comunicación a través de líneas editoriales opuestas (González et al. 2010). En este sentido no existe una producción académica muy fecunda que respalde la relación entre medios y reformas educativas. La poca literatura sobre el tema ha tendido a confirmar la idea general según la cual los medios construyen y transmiten una imagen negativa de la educación (Runte 2016), señalando a los gobiernos como responsables directos.

La polarización política ha sido un objeto de investigación por parte de múltiples académicos quienes, desde una aproximación multidisciplinar, han tratado de abordar este fenómeno social. Como ejemplo podemos destacar las diversas acepciones expuestas por Bramson et al. (2017), o el medidor geométrico-dicotómico de Poole (2008) de los años 80 del pasado siglo. Todos señalan que la polarización se genera cuando se produce una posición en favor de determinadas creencias, eludiendo su contrastación (Olsson 2013; Mason 2014).

El análisis de la disensión entre polos también está presente en los estudios de comunicación política (véase, por ejemplo, la excelente contribución de Sunstein 2002), especialmente en relación con los medios de comunicación (Prior 2013) y las perspectivas políticas (Gentzkow 2016). Por lo general, la polarización mediática puede interpretarse como la cobertura ideológicamente parcial y divergente de los medios (DiMaggio et al. 1996), aunque la mayoría de los estudios previos no ofrecen una definición operativa explícita del concepto (exceptuando el reciente trabajo de Boxell et al. 2020) puesto que, al menos a nivel más básico, se concibe como una noción de naturaleza suficientemente clara (Fletcher y Jenkins 2019). Sin embargo, la polarización también se ha definido como un efecto mediático o incluso un efecto político (Bernhardt et al. 2008). Durante los últimos años, los académicos han encontrado múltiples pruebas de que, en determinadas esferas mediáticas, no solo en los medios de comunicación convencionales sino también en las redes sociales y el entorno general de Internet, se puede producir un aumento de, y en, la polarización política o ideológica (Tucker et al. 2018), viéndose auspiciado por una sobreexposición, directa o indirecta, de nuestro modo y forma de consumir medios (De Vries 2020). No obstante, los vínculos potenciales que cabrían esperar entre la cobertura partidista de los medios de comunicación y la creciente fragmentación 
política de la ciudadanía no parecen haberse visto sustentados por una extensa evidencia empírica, aunque el potencial en este sentido es señalado de forma generalizada (Fletcher y Jenkins 2019).

La mayoría de los estudios que han abordado la polarización en la cobertura mediática, que además de no ser tan numerosos, no proponen una aproximación empírica contundente, habiéndose focalizado especialmente en los medios estadounidenses (Lowry y Shidler 1998; McCluskey y Kim 2012) o en temas que generan polarización intrínsecamente, particularmente tras determinados eventos llamativos para la opinión pública (Aruguete y Calvo 2018). En este sentido destacan, por ejemplo, los análisis sobre inmigración (Benson 2010; Balch y Balabanova 2011) o corrupción (Mancini et al. 2017). Se puede percibir un aumento de la investigación sobre polarización en las redes sociales en detrimento de los medios tradicionales (Tucker et al. 2018; Boxell et al. 2020).

A tal efecto, puede argumentarse que la política educativa es un tema que, por su propia naturaleza, induce a la polarización. En el caso concreto de España, por ejemplo, es esperable que los periódicos más cercanos a las ideologías conservadoras destaquen los aspectos negativos de los resultados de la política educativa socialdemócrata. Mientras, aquellos que defienden otros valores, como la equidad y la igualdad de acceso a la escuela, encuadran de forma desfavorable las reformas emprendidas por el partido conservador (Runte 2016: 723).

El objetivo de este artículo, entonces, es el de valorar, aplicando una metodología novedosa, el grado de polarización en los medios de comunicación escritos en relación con las reformas educativas que se han llevado a cabo en España desde la transición a nuestros días. Tras este proceso, podremos verificar el grado de paralelismo político (alineamiento ideológico) mostrado por los medios de comunicación de referencia siguiendo la clasificación de Hallin y Mancini (2004). Los medios de comunicación analizados son los diarios ABC y El País. Mientras que el primero cuenta con una línea conservadora y tradicional, que evoluciona hacia postulados más liberales en la actualidad, el segundo destaca por un posicionamiento más progresista, alineándose en torno ideales socialdemócratas (Humanes 2014). Lo que se espera es que la contraposición mediática, revelada en la cobertura (coincidiendo con los periodos de reformas educativas iniciadas por los partidos políticos ideológicamente opuestos), muestre cotas de dispersión (polarización), sirviendo éstas para engrasar y apuntalar el sistema y las instituciones (Kennamer 1994; Aruguete 2013; Martínez et al. 2014).

\section{DISEÑO Y MUESTRA}

Las hipótesis de la investigación están basadas, siguiendo los postulados teóricos anteriores, en la ideología como predictor del comportamiento mediático. Así, establecemos que: 
H1: La visibilidad (cantidad) de la cobertura mediática (salience) estará relacionada con la ideología del medio de comunicación.

H2: Los subtemas con los que se aborda dicha cobertura divergen de acuerdo a las diferencias ideológicas.

H3: La cobertura mediática tenderá a una mayor polarización cuando la línea editorial del medio sea opuesta a la ideología del partido en el gobierno.

En resumen, la cobertura entre los dos medios de comunicación analizados (ABC y El País) debería mostrar diferencias significativas y mensurables en la cantidad de artículos dedicados a las reformas educativas, pero también con respecto a los temas asociados (Miller 1997) a dichas reformas, y al tono o polarización de estas. Asimismo, cabría esperar que dichas diferencias se deban a la distancia ideológica de los diarios con respecto al partido del gobierno en ese momento.

La finalidad de esta propuesta es verificar las hipótesis planteadas abarcando todo el desarrollo democrático reciente de España, es decir, los últimos cuarenta años. Por ello, se han seleccionado los dos diarios de tirada nacional más representativos del periodo, siendo, a su vez, los únicos que abarcan toda la muestra temporal de estudio.

El corpus se conformó mediante una búsqueda por palabras clave parametrizadas mediante la horquilla temporal: 6 de diciembre de 1978 a 6 de diciembre de 2018, en la que se tomó como punto de partida el referéndum de ratificación de la Constitución española y, como cierre, su conmemoración 40 años después. Las palabras clave empleadas fueron «reforma educativa» y «ley de educación». El segundo conjunto de palabras fue añadido ya que, al iniciarse la búsqueda en primera instancia, se mostraban diferencias muestrales entre uno y otro medio. Este cambio a la hora de añadir un nuevo conjunto se podría tornar sintomático en la medida en que, los temas empleados, podrían convertirse en una variable significativa. Esto podría tenerse en cuenta para la confección y tratamiento de los artículos (se han incluido piezas de información y opinión). Las palabras clave al ser buscadas podrían encontrarse tanto en el título, subtítulo o cuerpo de la noticia. El total de esta muestra fue elaborado mediante el uso de las hemerotecas online de ABC y El País (1978-1995) y la base de datos MyNews ${ }^{13}$ (1996-2018). Mientras que los artículos obtenidos de la hemeroteca de ABC fueron conseguidos y conformados de forma manual, los correspondientes a El País se obtuvieron a través de un modelo de producción propia mediante la técnica de scraping. De este modo, y después de depurar la muestra y eliminar aquellos artículos que no trataban sobre reformas educativas concernientes al ámbito español, se obtuvo un total de 4872 artículos (2477 ABC y 2395 El País, un número asombrosamente similar para ambos medios a lo largo de cuarenta años). 
Una vez adquirido el corpus, el texto se procesó siguiendo los estándares en este tipo de estudios (eliminación de símbolos atípicos, eliminación de espacios en blanco, tokenización, etc., Feinerer et al. (2008), muestran las diferentes estrategias para el procesamiento de textos en $R$ (lenguaje que usamos en esta investigación) para, posteriormente, ser testeado en dos fases (más allá de las meras frecuencias). En primer lugar, se realizaron puntajes previos por medio de un análisis de sentimiento, con el fin de calificar la muestra. El proceso implementado se denomina SentimentR (Rinker 2019), y realiza un cálculo de la ambivalencia por contexto, donde se dejan a un lado los modelos clásicos por unidades de palabras (frecuencias) y se implementa un sistema contextual en el que se tienen en cuenta los cambios de valencia. Se busca, así, no distorsionar en gran medida la semántica ya que, en los análisis donde la partícula es la palabra, no se recoge una valoración íntegra, soslayando recursos del lenguaje como las expresiones retóricas. Esto propicia que la ambigüedad no sea captada en toda su riqueza y se confeccione una calificación más literal.

No obstante, como ese tipo de análisis no aporta más información que una declaración sobre lo positivo o negativo de las palabras o frases, hemos implementado una forma de medir la polarización (véase Serrano-Contreras et al. 2020 para una explicación más pormenorizada). Para ello, se operacionaliza la polarización de un artículo $\left(\mathrm{p}_{\mathrm{c}}\right.$ ) como la distancia entre el análisis de sentimiento de ese artículo $(S)$ y la mediana del agregado de los análisis de sentimiento de todos los artículos para ese diario $(\mathrm{Me})$, en número absoluto. De esta forma, se obtiene un número que puede tomar cualquier valor entre 0 y 2, donde 0 muestra que no hay polarización (aunque pueda mostrarse un sentimiento tanto positivo como negativo) y 2 muestra una polarización máxima. El ratio que establece nuestro índice tiene la capacidad de medir las posibles divergencias que, dentro de un arco temporal, pueden generarse en un medio.

$$
\mathrm{P} c=|(\mathrm{S}-\mathrm{Me})| \text {, donde } \mathrm{S} \in[-1,1], p \in(0,2)
$$

Complementariamente a lo anterior, y debido al número de artículos extraídos, hemos juzgado necesaria la aplicación de una técnica de extracción y reducción de la información contenida en la muestra. De esta forma podemos reducir la complejidad temática contenida en casi cinco mil artículos de periódico a unos pocos temas o ideas presentes en la mayoría de ellos. A este respecto, existen multitud de técnicas existentes, desde algoritmos supervisados (véase, por ejemplo, García-Marín y Calatrava 2018) hasta los no supervisados (como los LDA (Latent Dirichlet Allocation) y LSI (Latent Semantic Indexing). Como la muestra recoge publicaciones durante más de 40 años, los algoritmos supervisados no parecen aconsejables, ya que el entrenamiento debería estar basado en todos los temas existentes (y para todas las épocas). Por ello, hemos decidido la aplicación de una técnica no supervisada, en concreto por un algoritmo LSI. Este algoritmo es una técnica enmarcada dentro del Procesamiento Natural del Lenguaje (NLP por sus siglas en inglés) que se basa en analizar las relaciones entre un conjunto de documentos y los términos que contienen (por lo que está 
basado en frecuencias) mediante la producción de conceptos relacionados con esos documentos y términos. El algoritmo utiliza los conjuntos de textos de nuestro corpus para producir como resultado una serie de temas presentes $(k)$, a través de la distancia vectorial entre las palabras y a partir de los documentos, en nuestro caso, los artículos periodísticos (una buena explicación de su funcionamiento y utilidad puede encontrarse en Letsche y Berry (1997), lo que muestra que es una técnica bastante consolidada).

\section{ANÁLISIS Y RESULTADOS}

Los datos analizados se extienden a lo largo de un marco temporal de 40 años en el que se suceden siete presidentes del gobierno bajo las siglas de tres partidos políticos distintos. De un total de 4872 artículos, 3012 han sido publicados en periodos socialistas, seguidos de 1759 en gobiernos populares y 101 durante los de la desaparecida UCD. ${ }^{14}$ Desde un punto de vista más pormenorizado destacan las legislaturas presididas por el presidente Rodríguez Zapatero, quien acapara casi la mitad de la muestra, seguido de Felipe González y José María Aznar, respectivamente. Como podemos apreciar en el gráfico 1, los datos revelan una más que evidente significación entre el número de artículos publicados y el partido que se encuentra en el gobierno. Es decir que, mientras el color del gobierno se aleja de la línea ideológica del medio, este aumenta el número de artículos publicados, evidenciándose de forma directa, y casi total, en todos los periodos analizados. Por consiguiente, lo que se demuestra, en línea con lo expuesto por Budge y Farlie (1983) o Helbling y Tresch (2011), es que las discrepancias ideológicas no se manifiestan al confrontar posturas sobre un mismo tema, sino que es a través del énfasis que se da a una determinada temática lo que propicia que la atención vire y los posicionamientos tiendan a alejarse.

A su vez, cabe destacar un dato singular: cuando en el artículo se emplea alguno de los dos temas utilizados para la búsqueda, «reforma educativa» $\mathrm{y}$ «ley de educación», el otro no aparece, ocurriendo esta convergencia sólo en el 2,38\% de las ocasiones. Algo que podría mostrar diferencias semánticas e ideológicas, aunque los datos no revelan posicionamiento ideológico alguno y se podría deber más a un simple relato de la cuestión, como refleja de forma clara el tramo temporal en torno a la ley de educación catalana (2009). En cualquier caso, parece que, efectivamente, la visibilidad del tema sí está relacionada con la diferencia ideológica entre el medio de comunicación y el partido en el gobierno, por lo que la primera hipótesis se confirma. Así se muestra en el periodo analizado, por ejemplo, en el último año -2018-, donde en los primeros meses, y con gobierno del Partido Popular, El País publica más que ABC. Tendencia que se invierte en la segunda mitad del año con la llegada al poder de Pedro Sánchez.

14 La UCD es una plataforma política creada por Adolfo Suárez en el año 1977 y disuelta en 1983 (Martínez 2017). A pesar de su corto periodo de existencia, la formación hizo las veces de correa de transmisión entre el viejo régimen franquista y la democracia que empieza a construirse en aquellos años. 
Gráfico 1. Agregado de artículos por año

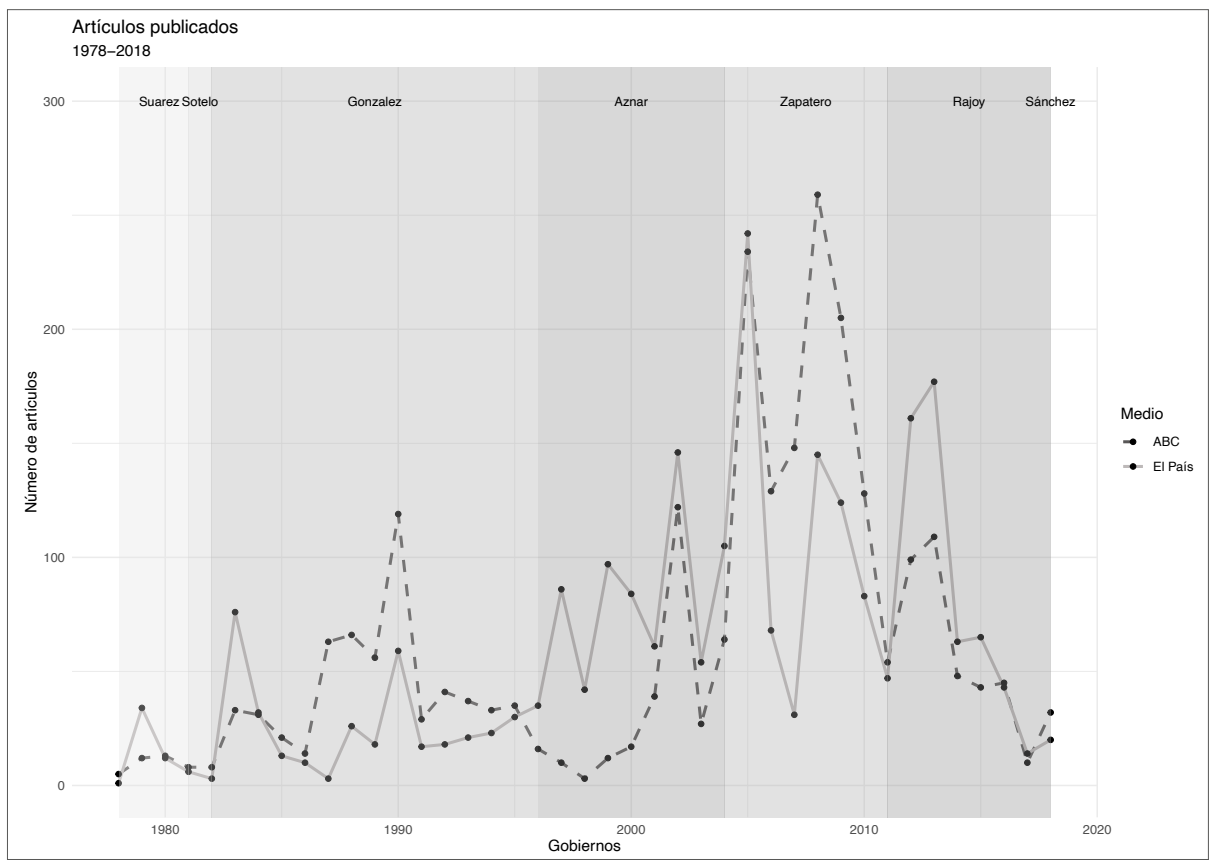

Elaboración propia. Nota sobre agregados $2018 .{ }^{15}$

Con respecto a la polarización de los medios de comunicación, el gráfico 2 muestra este parámetro de forma cronológica y contextual. Como se puede apreciar, el índice parece exponer de manera fiel cómo se produce una dispersión temporal entre medios y gobiernos. Si bien, a lo largo del periodo de análisis, la muestra se va comportando de manera diferente, demostrando que el índice al menos es capaz de mostrar variaciones consistentes con la realidad observable y, además, a nuestro parecer, de forma adecuada. A pesar de ello, no se puede asegurar que las altas cotas de polarización sean una constante dentro de los periodos de análisis, aunque sí se destilan ciertos patrones en los que los medios tienden a polarizar su cobertura en función del partido que se encuentra en el poder. Sin embargo, es importante tener presente que la polarización no indica la dirección de la misma; es decir, entendemos que la ideología es un predictor de la polarización, pero ésta puede estar motivada por el propio debate político y mediático, por lo que el apoyo a una determinada reforma educativa podría hacerse también desde posturas polarizantes, sobre todo si se producen ataques a la misma por parte de la oposición.

15 Nota sobre agregados 2018. Durante el año 2018 hubo un cambio en la presidencia del Gobierno, produciendo de forma desagregada: Rajoy [ABC: 9 - El País: 11] y Sánchez [ABC: 24 - El País: 8] 
Gráfico 2. Polarización El País y ABC (1978-2018)

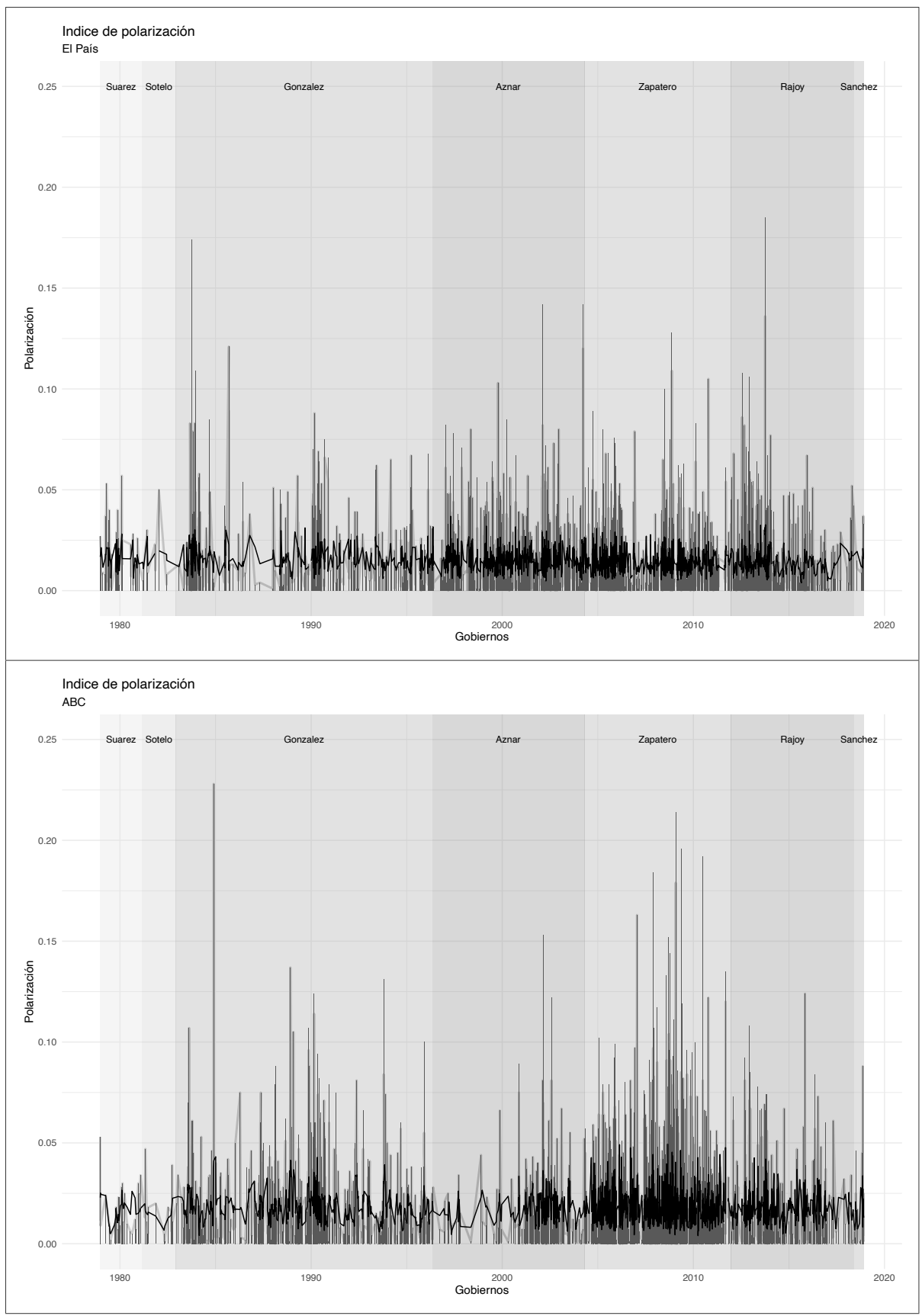

Elaboración propia. La línea oscura representa la media móvil diaria.

Por último, dentro de los conjuntos de análisis llevados a cabo se encuentra la aplicación de un algoritmo no supervisado, en concreto, un LSI. Este modelo 
de descomposición semántica mediante vectorización (Hoffmann 1999) señala la variabilidad de subtemas que esta temática provoca en los dos periódicos, tal y como reflejan las tablas 1 y 2 . La tabla 1 muestra la cobertura de El País para el período de análisis. Naturalmente, los temas seleccionados tienen que ser, debido al tamaño de la muestra, forzosamente genéricos. Sin embargo, dos elementos parecen importantes: primero, que las referencias al «partido popular» han sido más o menos constantes en el período, estando presentes en tres de los temas; y, segundo, que el quinto tema parece estar dedicado a los aspectos territoriales y lingüísticos de las leyes de educación.

Tabla 1. Análisis de la cobertura de El País (1978-2018) ${ }^{16}$

\begin{tabular}{ccc}
\hline Tema & Términos presentes & Términos ausentes \\
\hline $\mathbf{1}$ & educación, ley, gobierno, alumnos, reforma, centros, pp & \\
$\mathbf{2}$ & educación, centros, alumnos, enseñanza & pp, gobierno \\
$\mathbf{3}$ & ley, gobierno, pp, proyecto & alumnos, años, \\
$\mathbf{4}$ & millones, pesetas, proyecto & educación \\
$\mathbf{5}$ & alumnos, pp, gobierno, castellano, cataluña, centros, curso & educación, ley, física \\
\hline
\end{tabular}

Nota: Los términos se han editado para mejorar la legibilidad. Términos seleccionados en negrita.

En cuanto a $\mathrm{ABC}$, en la tabla 2, observamos un comportamiento parecido a El País, aunque con diferencias significativas, tal y como establecía la H2. Las semejanzas se producen en la presencia de términos como «partido popular» o el tema lingüístico. Pero, en este caso, aparece el tema religioso, ausente de los principales elementos de la cobertura de El País, lo que no es sorprendente pero sí indicativo de diferentes formas de enfocar las reformas educativas desde presupuestos ideológicos y de valores opuestos. Lo mismo puede decirse del término "padres», sólo presente en ABC. Es decir, el algoritmo ha sido capaz de mostrar diferencias consistentes en la cobertura mediante los diferentes enfoques que cada medio otorga a la realidad informativa (sobre esos enfoques se puede ver Canel 1999).

16 Nota aclaratoria de las Tabla 1 y 2. Los términos seleccionados se incluyen con los estándares de limpieza, homogeneización y tokenización propios de este tipo de análisis. Por ello, se pueden encontrar tokens sin acento o aquellos otros que deberían de ir con la mayúscula inicial en minúscula. 
Tabla 2. Análisis de la cobertura de ABC (1978-2018)

\begin{tabular}{|c|c|c|}
\hline Tema & \begin{tabular}{|l} 
Términos presentes \\
\end{tabular} & Términos ausentes \\
\hline 1 & $\begin{array}{c}\text { educación, ley, enseñanza, reforma, gobierno, centros, } \\
\text { alumnos, educativa }\end{array}$ & \\
\hline 2 & gobierno, pp, españa & $\begin{array}{l}\text { educación, enseñanza, } \\
\text { centro, alumnos, formación }\end{array}$ \\
\hline 3 & ley, educación, gobierno, pp, catalán & reforma, formación \\
\hline 4 & años, curso, alumnos & $\begin{array}{l}\text { enseñanza, ley, proyecto, } \\
\text { derecho, libertad, religión }\end{array}$ \\
\hline 5 & padres, centros, alumnos, enseñanza, iglesia & $\begin{array}{l}\text { reforma, educación, } \\
\text { proyecto, formación }\end{array}$ \\
\hline
\end{tabular}

Nota: Los términos se han editado para mejorar la legibilidad. Términos seleccionados en negrita.

Pero, ¿cuál es la relación entre la polarización y los temas tratados por los medios de comunicación? A este respecto, las tablas 3 y 4 muestran la matriz de correlaciones entre las variables de análisis (nótese que los temas varían en contenido para cada medio). La primera conclusión que se puede extraer es que las correlaciones son débiles en todos los casos falsando, en principio, la última hipótesis (H3), ya que no se aprecia un aumento o descenso significativo entre polarización y partido en el gobierno.

En concreto, hay dos temas que sobresalen en la matriz: Cataluña (tema 3) y la religión (tema 5). En los dos casos observamos comportamientos divergentes en la cobertura mediática. Es decir, los resultados del análisis siempre son inversos en todos los temas, aunque no sean demasiado significativos en algunos casos. Esto resulta interesante porque, en principio, se esperaba que el comportamiento con respecto a los temas, aunque no fuera exactamente igual (H2), sí que estuviera tratado en ambos medios de comunicación, especialmente porque la iniciativa en la exposición de los temas debería ser gubernamental o parlamentaria. Sin embargo, los resultados parecen apoyar la idea de que los temas no dependen del nivel político, sino que estarían más relacionados con la agenda mediática. Y esto puede suceder de dos formas diferentes, desde nuestra perspectiva: por un lado, siguiendo los postulados de los teóricos de los estudios de la construcción de la agenda (el estudio más popular, clásico y descriptivo de la teoría puede ser el de Cobb et al. 1976), se podría considerar que los medios de comunicación centran el debate en los temas que consideran más interesantes o propicios (es decir, muestran los temas usando los encuadres que estimen); por otro lado, otros académicos han afirmado que los medios de comunicación se encuentran indexados al nivel político, reflejando el nivel de desacuerdo entre los grupos $\mathrm{y}$, habitualmente, tomando partido por un lado u otro (Bennett 2016). La diferencia entre ambos reside en el origen de la información. Mientras que, en el primer caso, los medios de comunicación tienen la capacidad y la voluntad de afectar al debate político proponiendo temas o encuadres específicos; en el segundo, se limitan a reflejar lo que los actores institucionales exponen. 
Los datos que mostramos aquí podrían apoyar ambos acercamientos y sólo un detallado estudio cronológico ayudaría a dilucidar la cuestión.

Tabla 3. Correlaciones El País -Rho de Spearman, términos seleccionados-

\begin{tabular}{ccccccc}
\hline Part_Gob & Polarización & Tema 1 & Tema 2 & Tema 3 & Tema 4 & Tema 5 \\
\hline PP & -0.025 & $.054^{* *}$ & -0.006 & $\mathbf{- . 1 9 6 * *}$ & $.107^{* *}$ &. $\mathbf{2 0 1}$ \\
PSOE & 0.01 & $-.056^{* *}$ & 0.003 & $\mathbf{. 1 9 5}^{* *}$ & $-.127^{* *}$ & $\mathbf{- . 1 5 2}^{* *}$ \\
UCD & .050 & 0.005 & 0.008 & 0.001 & $.065^{* *}$ & $\mathbf{- . 1 6 4 ^ { * * }}$ \\
\hline
\end{tabular}

** La correlación es significativa en el nivel 0.01 (bilateral).

* La correlación es significativa en el nivel 0.05 (bilateral).

Tabla 4. Correlaciones ABC -Rho de Spearman, términos seleccionados-

\begin{tabular}{ccccccc}
\hline Part_Gob & Polarización & Tema 1 & Tema 2 & Tema 3 & Tema 4 & Tema 5 \\
\hline PP & $-.055^{* *}$ & $-.074^{* *}$ &. $\mathbf{1 3 8 ^ { * * }}$ & $-.128^{* *}$ & $.118^{* *}$ & $-.063^{* *}$ \\
PSOE & $.051^{*}$ & $.077^{* *}$ & $\mathbf{- . 1 4 8 ^ { * * }}$ & $.148^{* *}$ & $-.123^{* *}$ & $.056^{* *}$ \\
UCD & 0.009 & -0.015 & .044 & $-.074^{* *}$ & 0.025 & 0.018 \\
\hline
\end{tabular}

** La correlación es significativa en el nivel 0.01 (bilateral).

* La correlación es significativa en el nivel 0.05 (bilateral).

Sea cual sea el motivo de las diferencias de cobertura entre los dos medios de comunicación (que, en todo caso, es débil), su mera existencia sí que podría confirmar que España es un caso de pluralismo polarizado, por su visible nivel de paralelismo político. Sin embargo, no sería a través de la polarización de la cobertura con respecto a los temas, sino tan sólo con respecto a la exposición de los mismos (a pesar de que el gráfico 2 sugiera que sí hay diferencias en la polarización).

\section{DISCUSIÓN Y RESULTADOS}

La meta principal de este estudio era verificar si las tesis de Hallin y Mancini (2004) se cumplen en el sistema mediático español, testando una propuesta metodológica novedosa en el campo de la comunicación política. Para ello se seleccionó un tema que tuviera un cariz polarizante dentro de la sociedad; la educación. Para focalizar esta temática decidimos centrar nuestra investigación en el empleo de las partículas semánticas «reforma educativa» y «ley de educación» a lo largo de los primeros cuarenta años de democracia en España. De este modo, tras implementar el diseño de investigación detallado, podemos afirmar que los resultados confirman en general las dos primeras hipótesis de partida, aunque no la tercera. 
En primer lugar, las observaciones arrojan una relación entre las líneas ideológicas de los medios y su posicionamiento con respecto a las reformas educativas tramitadas por parte de los partidos en el poder, tal y como se aprecia de forma muy clara en las figuras de agregados por artículos. Se establece con ello una clara interrelación entre el número de artículos, que incluyen cualquiera de los topics de búsqueda, y el partido de gobierno. Consecuentemente, se evidencia que la cantidad de artículos es mayor en función de si la línea editorial del periódico se distancia de los preceptos ideológicos del partido que pretende promulgar la ley.

En segundo lugar, y con las evidencias expuestas, se procedió a completar esta investigación con un modelo de aprendizaje de máquina automatizado, LSI (Latent Semantic Indexing). Este proceso nos permitió ver cómo era la cobertura y en qué temáticas solían centrarse, y divergir los dos periódicos a la hora de hablar de educación. Debido a la magnitud de la muestra, hay temas que sobresalen, por comunes y sintomáticos, a la cuestión de estudio, y hay otros significantes por los que se hallan diferencias entre medios. Por ejemplo, en el caso de El País, las cuestiones "Cataluña» y "castellano» suponen una vertiente de gran interés para el medio, mientras que, en el caso de $\mathrm{ABC}$, lo que se encontraría es una prenoción por «catalán», así como por el de «iglesia», o la importancia que se suscita entre el tema educativo y «padres». Esto muestra las distancias que se generan a la hora de abordar los temas por los medios y que, en el caso analizado, abre discrepancias en cuanto a los intereses que llevan a uno y a otro a hablar de educación.

Por último, las técnicas se han ido complementando con el fin de mostrar los resultados y, a su vez, servir de campo de pruebas para futuros estudios. En este sentido, se ha buscado desarrollar nuevas líneas de actuación que sirvan para medir la polarización dentro de distintos escenarios de opinión pública, desde medios de comunicación, hasta redes sociales. El índice empleado ha mostrado un gran rendimiento (véase Serrano et al. (2020) como ejemplo), siendo capaz de captar de un modo más complejo, la frecuente simplicidad semántica que arrojaran las valencias utilizadas para el análisis de sentimiento.

A pesar de ello, todavía existen dificultades para detectar un vocabulario más discrepante, como puede ser el empleado en las redes sociales. Este hecho, propicia que el equilibrio se acabe moderando debido a los subconjuntos semánticos que componen un texto periodístico. Es más, la polarización mostrada por la herramienta no incluye la dirección de la misma; es decir, los dos medios de comunicación polarizan en ciertos momentos su cobertura, especialmente con respecto a la línea ideológica del gobierno (lo que se aprecia en el gráfico 2), pero no lo suficiente como para detectar un patrón (de ahí la falta de significación de las tablas 3 y 4). Y, seguramente, si se analiza hacia dónde va dirigida esa polarización (el gobierno o la oposición) sí se encuentre ese último eslabón que aquí sólo se intuye, por lo que se debe virar hacia técnicas de localización más sofisticadas (a saber, Ghatak 2019; Hodnett et al. 2019; Rezaeinia et al. 2019). 
Hasta el momento, ha parecido inviable un pacto entre los partidos políticos que se alternaron en el poder, PSOE y PP, como entre los bloques ideológicos que han dividido el Parlamento español en las últimas décadas. El calado de las reformas en algunos aspectos no distan mucho, pero existen dos elementos que serán clave a la hora de polarizar a los actores políticos y sociales: la iglesia católica y la privatización del sector educativo (Viñao 2006: 59). Y esto, en un sistema multinivel como el español, genera un efecto multiplicador en las pulsiones polarizantes, ya que cada reforma ha sido dificultada y desactivada hasta lo posible por las comunidades autónomas con gobiernos de ideología distinta al impulsor de la reforma a nivel central. España todavía carece de una tradición consociacional democrática, donde la educación no se considera una moneda de cambio y sí una piedra angular para el desarrollo del país. Parece que alcanzar un pacto de estado en esta materia se interpreta como asumir unos costes demasiado altos sin ningún -o pocos- beneficio, y que hacerlo supondría ignorar las preferencias de sus votantes. Pactar en política educativa equivaldría, pues, a entregarse imperdonablemente al enemigo (Gortázar y Moreno 2017).

Esta investigación abre nuevas vías y enfoques para el avance dentro de la comunicación política, y concretamente para el análisis de las influencias que el sector político ejerce en los distintos ámbitos de la esfera pública. Con este trabajo se pretende ofrecer un primer paso para el contraste evolutivo de esta temática, que se vuelve a mostrar en constante vigencia hoy día, especialmente vinculado a la transformación que ha sufrido el arco parlamentario hacia la fragmentación. Este cambio, junto con el primer ensayo de gobierno de coalición en España en 2020, muestra una nueva veta de investigación que podría alterar los patrones mostrados en el presente estudio. Por lo tanto, observando los movimientos de los grupos parlamentarios tras la formación del presente gobierno, en relación con la reforma de la ley educativa vigente, podríamos plantearnos si los paradigmas contrastados en este proyecto seguirán siendo la tónica o, si por el contrario, se producirán pactos de gran calado entre las más que complejas amalgamas ideológicas que, a su vez, se proyecten a la esfera mediática.

Finalmente, nos gustaría señalar que el empleo creciente de nuevas técnicas de investigación automatizadas es un hecho. Si bien el desarrollo y la proliferación de su uso se ha producido inicialmente en otras disciplinas más técnicas, en los últimos años hemos observado que investigaciones propuestas desde las ciencias sociales están incluyendo estas herramientas en su diseño. Asimismo, cabe destacar que este proceso está impulsado tanto por la facilidad del acceso a datos estructurados (hasta ser prácticamente ilimitados), como la generación de procesos para estandarizar la información para el análisis. 


\section{REFERENCIAS}

Arriagada, Arturo, Patricio Navia y Martín Schuster. 2010. “¿Consumo Luego Pienso, O Pienso Y Luego Consumo?: Consumo de Medios, Predisposición Política, Percepción Económica Y Aprobación Presidencial en Chile." Revista de Ciencia Política 30(3): 669-695.

Aruguete, Natalia. 2013. "La Narración Del Espectáculo Político: Pensar la Relación Entre Sistema de Medios Y Poder Político." Austral Comunicación 2(2): 205-216.

Aruguete, Natalia y Ernesto Calvo. 2018. "Time to \#Protest: Selective Exposure, Cascading Activation, and Framing in Social Media." Journal of Communication 68(3): 480-502.

Balch, Alex y Ekaterina Balabanova. 2011. "A System in Chaos? Knowledge and Sense-Making on Immigration Policy in Public Debates." Media, Culture E Society 33(6): 885904.

Balkin, Jack M. 1999. "How Mass Media Simulate Political Transparency." Cultural Values 3(4): 393-413.

Bennett, W. Lance, Victor Pickard, David Iozzi, Carl Schroeder, Taso Lagos y C. Evans Caswe11. 2004. "Managing the Public Sphere: Journalistic Construction of the Great Globalization Debate." Journal of Communication 54(3): 1437-455.

Bennett, W. Lance. 2016. "Indexing Theory." En The International Encyclopedia of Political Communication, editado por Gianpietro Mazzoleni. Hoboken: Wiley.

Benson, Rodney. 2010. "What Makes for a Critical Press? A Case Study of French and U.S. Immigration News Coverage." The International Journal of Press/Politics 15(1): 3-24.

Bernhardt, Dan, Stefan Krasa y Mattias Polborn. 2008. "Political Polarization and the Electoral Effects of Media Bias." Journal of Public Economics 92(5-6): 1092-1104.

Boxell, Levi, Matthew Gentzkow y Jess Shapiro. 2020. "Cross-Country Trends in Affective Polarization." Working Paper No. 26669. National Bureau of Economic Research.

Bramson, Aaron, Patrick Grim, Daniel Singer, William Berger, Graham Sack, Steven Fisher, Carissa Flocken y Bennett Holman. 2017. "Understanding Polarization: Meanings, Measures, and Model Evaluation." Philosophy of Science 84(1): 115-159.

Budge, Ian y Dennis Farlie. 1983. "Party Competition: Selective Emphasis or Direct Confrontation? An Alternative View With Data." En Western European Party Systems: Continuity $\mathcal{E}$ Change, editado por Hans Daalder y Peter Mair. London: SAGE Publications, 267-305.

Canel, María. 1999. "El País, ABC y El Mundo: Tres Manchetas, Tres Enfoques de Las Noticias." ZER - Revista de Estudios de Comunicación 1(6):1-11.

Cobb, Roger, Jennie-Keith Ross y Marc Howard. 1976. "Agenda Building as a Comparative Political Process." American Political Science Review 70(1): 126-138.

Collins, Todd y Christopher Cooper. 2012. "Case Salience and Media Coverage of Supreme Court Decisions: Toward a New Measure." Political Research Quarterly 65(2): 396-407.

De Vries, Gerdien. 2020. "Public Communication as a Tool to Implement Environmental Policies." Social Issues and Policy Review 14(1): 244-272.

DiMaggio, Paul, John Evans y Bethany Bryson. 1996. "Have American's Social Attitudes Become More Polarized?" American Journal of Sociology 102(3): 690-755.

Entman, Robert. M. 1993. "Framing: Toward Clarification of a Fractured Paradigm." Journal of Communication 43(4): 51-58.

Feinerer, Ingo, Kurt Hornik y David Meyer. 2008. "Text Mining Infrastructure in R." Journal of Statistical Software 25(5): 1-54.

Fletcher, Richard y Joy Jenkins. 2019. Polarisation and the News Media in Europe. Bruselas: EPRS.

García-Marín, Javier y Adolfo Calatrava. 2018. "The Use of Supervised Learning Algorithms in Political Communication and Media Studies: Locating Frames in the Press." Comunicación y Sociedad 31(3): 175-188.

Gentzkow, Matthew. 2016. Polarization in 2016. Mimeo: Stanford University.

Ghatak, Abhijit. 2019. Deep Learning with R. Singapur: Springer.

Gil, Daniel, Carlos Furió y Valentín Gavidia. 1998. “El Profesorado y la Reforma Educativa en España." Revista Investigación en la Escuela (36): 49-64. 
González, Juan Jesús y Fermín Bouza. 2009. Las Razones Del Voto en la España Democrática (1977-2008). Madrid: Catarata.

González, Juan Jesús, Raquel Rodríguez y Antón Rodríguez. 2010. “A Case of Polarized Pluralism in a Mediterranean Country. The Media and Politics in Spain." Global Media Journal Mediterranean Edition, $5(1 / 2)$

Gortázar, Lucas y Juan Moreno. 2017. “Costes y Consecuencias de no Alcanzar un Pacto Educativo en España." Revista Educación, Política y Sociedad 2(2): 9-37.

Hallin, Daniel y Paolo Mancini. 2004. Comparing Media Systems Three Models of Media and Politics. Nueva York: Cambridge University Press.

Helbling, Marc y Anke Tresch. 2011. "Measuring Party Positions and Issue Salience From Media Coverage: Discussing and Cross-Validating New Indicators." Electoral Studies 30(1): 174-183.

Hodnett, Mark, Joshua F. Wiley, Yuxi Luiu y Pablo Maldonado. 2019. Deep Learning with $R$ for Beginners. Birmingham: Packt.

Hoffman, Thomas. 1999. "Probabilistic Latent Semantic Analysis." Ponencia presentada en Proceedings of Uncertainty in Artificial Intelligence, Estocolmo, 30 de julio a 1 de agosto.

Humanes, María L. 2014. "Exposición Selectiva y Partidismo de las Audiencias en España. El Consumo de Información Política Durante las Campañas Electorales de 2008 y 2011." Palabra Clave 17(3): 773-802.

Humanes, María L., María D. Montero, Ramón Molina y Alfredo López-Berini. 2013. “Pluralismo y Paralelismo Político en la Información Televisiva en España." Revista Latina de Comunicación Social 68(5): 24-16.

Kennamer, David. 1994. Public Opinion, the Press, and Public Policy. London: Praeger.

Letsche, Todd y Michael W. Berry. 1997. "Large-Scale Information Retrieval with Latent Semantic Indexing." Information sciences 100(1-4): 105-137.

Lodola, Germán y Philip Kitzberger. 2017. "Politización y Confianza en los Medios de Comunicación: Argentina Durante el Kirchnerismo." Revista de Ciencia Política 37(3): 635658.

Lowry, Dennis y Jon A. Shidler. 1998. "The Sound Bites, the Biters, and the Bitten: A Two-Campaign Test of the Anti-Incumbent Bias Hypothesis in Network TV News." Journalism E Mass Communication Quarterly 75(4): 719-729.

Mancini, Paolo, Marca Mazzoni, Alessio Cornia y Rita Marchetti. 2017. "Representations of Corruption in the British, French, and Italian Press: Audience Segmentation and the Lack of Unanimously Shared Indignation." The International Journal of Press/Politics 22(1): 67-91.

Martínez, Juan B. 2017. “¿Y Ahora Qué? Impacto de la Disolución de la UCD en El Mapa Político Local de las Provincias de Albacete y Murcia." En Los Embates de la Modernidad: Debates en Torno a la Ciudadanía, El Liberalismo, El Republicanismo, la Democracia y Los Movimientos Sociales, editado por Oriol Luján y Laura Canalias Chorrero. Barcelona: DHMC, 563-579.

Martínez, Manuel, María L. Humanes y Enric Saperas. 2014. “La Mediatización de la Política en El Periodismo Español. Análisis Longitudinal de la Información Política en la Prensa de Referencia (1980-2010)." Tripodos (34): 41-59.

Mason, Lilliana. 2014. "“I Disrespectfully Agree”: The Differential Effects of Partisan Sorting on Social and Issue Polarization." American Journal of Political Science 59(1): 128-145.

McCluskey, Michael y Young Kim. 2012. "Moderatism or Polarization? Representation of Advocacy Groups' Ideology in Newspapers." Journalism \& Mass Communication Quarterly 89(4): 565-584.

Miller, Mark. 1997. "Frame Mapping and Analysis of News Coverage of Contentious Issues." Social Science Computer Review 15(4): 367-378.

Monarca, Héctor, Cecilia Simón, Soledad Rappoport y Gerardo Echeita. 2016. "Política y Cambio en Educación: El Caso de las Competencias Básicas en España." Ensaio: Avaliação e Políticas Públicas em Educação 24(93): 968-989. 
Olsson, Erik J. 2013. "A Bayesian Simulation Model of Group Deliberation and Polarization." En Bayesian Argumentation Synthese Library (Studies in Epistemology, Logic, Methodology, and Philosophy of Science) vol 362, editado por Frank Zenker. New York: Springer Publishing, 113-133.

Poole, Keith. 2008. "Las Raíces de la Polarización de la Política Moderna en los Estados Unidos." Revista de Ciencia Política 28(2): 3-37.

Prior, Markus. 2013. "Media and Political Polarization." Annual Review of Political Science 16(1): 101-127.

Rezaeinia, Seyes M., Rouhollah Rahmani, Ali Ghodsi y Hadi Veisi. 2019. "Sentiment Analysis Based on Improved Pre-Trained Word Embeddings." Expert Systems with Applications 117: 139-147.

Rinker, Tyler. 2019. “Trinker/Sentimentr." Recuperado el 23 de agosto de 2019 de https:/ / bit.ly/2VBwWBK

Runte, Ariadne 2016. "Pisa en la Prensa Española y su Influencia sobre las Políticas Educativas." Opción 32(8): 713-733.

Serrano-Contreras, Ignacio-Jesús, Javier García-Marín y Óscar Luengo. 2020. "Measuring Online Political Dialogue: Does Polarization Trigger More Deliberation?" Media and Communication 8(4): 63-72.

Sunstein, Cass. 2002. "The Law of Group Polarization." Journal of Political Philosophy 10(2): 175-195.

Tucker, Joshua, Andrew Guess, Pablo Barberá, Cristian Vaccari, Alexandra Siegel, Sergey Sanovich, Denis Stukal y Brendan Nyhan. 2018. Social Media, Political Polarization, and Political Disinformation: A Review of the Scientific Literature. Menlo Park: Hewlett Foundation.

Viñao, Antonio. 2006. “El Éxito O Fracaso de las Reformas Educativas: Condicionantes, Limitaciones, Posibilidades." En La Reforma Necesaria: Entre la Política Educativa y la Práctica Escolar, editado por José Gimeno Sacristán. Madrid: Ediciones Morata, 43-60.

Recibido: 9 de setiembre de 2020

Aceptado: 18 de abril de 2021

Ignacio-Jesús Serrano-Contreras es PhD Candidate en Ciencias Políticas en la Universidad de Granada (Escuela de Doctorado de Ciencias Sociales). Sus líneas de investigación están focalizadas en la polarización, el feminismo y los usos e influjos de los medios de masas. Actualmente trabaja en el desarrollo e implementación de algoritmos como metodología para medir los fenómenos sociales. Ha sido Investigador Visitante en la Universidad de Hildesheim (Alemania), la Universidad de Surrey (Reino Unido), la Universidad de Bergen (Noruega) y la Universidad de Mostar (Bosnia). E-mail: nachosc@correo.ugr.es

Javier García-Marín es Profesor Titular en el Departamento de Ciencias Políticas y Administración Pública en la Universidad de Granada. Es PhD en Ciencias Políticas y Máster en Economía y Relaciones Internacionales. Ha sido Profesor Visitante en la London School of Economics and Political Science, la Universidad de Glasgow (Glasgow Media Unit), en la Amsterdam School for Communications Research, en el Institut d'Études Politiques de Bordeaux y en la Universidad de Buenos Aires. Además ha formado parte de varios proyectos internacionales. E-mail: javiergmarin@ugr.es

Óscar G. Luengo es Profesor Titular en el Departamento de Ciencias Políticas y Administración Pública en la Universidad de Granada. Es Co-Chair del Research Committee on Political Communication (RC22) de la International Association of Political Science (IPSA). Director de la Escuela Iberoamericana de Altos Estudios en Gobierno Local de la Unión Iberoamericana de Municipalistas (UIM). Ha sido Investigador Visitante en la European University Institute (Florencia, Italia), en la Johannes Gutenberg-Universität (Mainz, Alemania), y en la Amsterdam School of Communications Research (Países Bajos). Profesor Visitante en la Universidad de California, Berkeley (EE.UU.) entre otras universidades. E-mail: ogluengo@ugr.es 